\title{
Estratificação do risco cardiovascular em idosos portadores de síndrome metabólica
}

\author{
Cardiovascular risk stratification in the elderly with metabolic syndrome \\ Estratificación del riesgo cardiovascular en idosos portadores de síndrome metabólico \\ Vinicio Ramalho Rodrigues ${ }^{1 *}$, Francisco de Assis França Rodrigues ${ }^{1}$, Janio Cipriano Rolim ${ }^{1}$, Milena \\ Nunes Alves de Sousa ${ }^{1}$, Theonys Diogenes Freitas ${ }^{1}$, Lucíola Abílio Diniz Melquiades de Medeiros Rolim¹.
}

\section{RESUMO}

Objetivo: Estratificar o risco cardiovascular dos pacientes idosos portadores da Síndrome Metabólica. Métodos: Trata-se de uma pesquisa quantitativa realizada através de uma amostra probabilística aleatória simples com 182 usuários idosos das Unidades Básicas de Saúde de uma cidade do interior da Paraíba, nos quais foram coletados dados sociodemográficos, antropométricos e laboratoriais bioquímicos. Os dados foram analisados mediante estatística descritiva simples, dos testes t, Qui-Quadrado, exato de Fisher e Kruskal Wallis para correlação de variáveis, diagnóstico de Síndrome Metabólica pelos critérios NCEP-ATPIII e a estratificação de risco cardiovascular em 10 anos segundo o Escore de Framingham. Resultados: A porcentagem de participantes diagnosticados como portadores de Síndrome Metabólica e classificados pelo Escore de Framingham foram 19,84 \% para baixo risco, 34,92\% para moderado risco e $45,23 \%$ para alto risco, desse último 40,59\% eram do sexo feminino. A presença da síndrome metabólica possuiu significância estatística em relação a distribuição do risco cardiovascular, ou seja, ser portador de síndrome metabólica aumentou 2,14 vezes o risco cardiovascular na população estudada, com nível maior para o sexo feminino (3,74 vezes). Conclusão: A população deste estudo apresentou risco cardiovascular elevado, além da relevante prevalência dos fatores de risco utilizados no Escore de Framingham e na classificação da Síndrome Metabólica.

Palavras-chave: Risco cardiovascular, Síndrome X metabólica, Idoso.

\begin{abstract}
Objective: To stratify the cardiovascular risk of the elderly patients with metabolic syndrome. Methods: This study is a quantitative study using a random simple probabilistic sample of 182 elderly users of the Basic Health Units of a city in the interior of Paraíba, in which was collected sociodemographic, anthropometric and biochemical laboratory. The data were analyzed using simple descriptive statistics, t Student teste, Qui-Square test, Fisher exact test e Kruskal Wallis test for correlation of variables, diagnosis of Metabolic Syndrome by the NCEP-ATPIII criteria and cardiovascular risk stratification in 10 years according to Framingham Score. Results: The percentage of participants diagnosed as having metabolic syndrome and classified by Framingham Score were $19.84 \%$ for low risk, $34.92 \%$ for moderate risk and $45.23 \%$ for high risk, of the latter, $40.59 \%$ were of the sex female. The presence of the metabolic syndrome was statistically significant for the distribution of the cardiovascular risk, increasing by 2.14 times for the general sample, with a higher level for the female sex (3.74x). Conclusion: The population of this study had a high cardiovascular risk, in addition to the relevant prevalence of the risk factors used in Framingham Score and metabolic syndrome classification.
\end{abstract}

Keywords: Cardiovascular risk, Metabolic syndrome, Eldery.

${ }^{1}$ Centro Universitário de Patos (UNIFIP), Patos - PB.

*E-mail: vinicio.ramalho.13@gmail.com

SUBMETIDO EM: 7/2020

ACEITO EM: 8/2020

PUBLICADO EM: 12/2020

REAS / EJCH | Vol.12(12) | e4596 | DOI: https://doi.org/10.25248/reas.e4596.2020 Página 1 de 9 


\section{RESUMEN}

Objetivo: Detallar el riesgo cardiovascular de los pacientes ancianos y portadores de la síndrome. Métodos: Se trata de una investigación cuantitativa y realizada a través de una muestra de probabilidad simple al azar con 182 pacientes de las Unidades Básicas de Salud de una ciudad del interior de Paraíba, en los cuales fueron colectados datos sociodemográficos, antropométricos y laboratoriales bioquímicos. Los datos se analizaron utilizando estadísticas descriptivas simples, la prueba t de Student, la prueba de Chi-cuadrado, la prueba exacta de Fisher y la prueba de Kruskal Wallis para la correlación de variables, diagnóstico del síndrome x metabólico por los criterios NCEP-ATPIII y estratificación del riesgo cardiovascular en 10 ãnos según el puntaje de Framingham. Resultados: El porcentaje de diagnóstico como portadores de síndrome metabolica y clasificados por el Escore de Framingham fueron 19,84\% de riesgo bajo, $34,92 \%$ de riesgo moderado y $45,23 \%$ de riesgo alto, de ese último $40,59 \%$ eran mujeres. La presencia de la síndrome fue significativa estadísticamente para la distribución del riesgo cardiovascular, aumentando en 2,14 veces para la muestra general, con nivel mayor para las mujeres (3,74 veces). Conclusión: Hubo riesgo cardiovascular elevado y un predominio considerable de los factores de riesgo utilizados en el Escore de Framingham y en la clasificación de la síndrome metabolica.

Palabras clave: Riesgo cardiovascular, Síndrome X metabólica, Anciano.

\section{INTRODUÇÃO}

O desenvolvimento socioeconômico e a industrialização, associada à urbanização dos grandes centros, ocorrido ao longo da história, proporcionou ao mundo moderno bem-estar e aumento da expectativa de vida. Assim, não é mais necessário que o homem gaste considerável energia para obter seu próprio alimento ou para se locomover. Esse conjunto de fatores, aliado a aspectos culturais e genéticos, proporcionou o aumento do sedentarismo e do consumo inapropriado de alimentos industrializados ricos em sódio, açúcares e gorduras saturadas que levaram ao aumento do sobrepeso e obesidade na população mundial (BARBALHO SM, et al., 2015; BORTOLETTO MSS, et al., 2016)

Segundo a Organização Mundial da Saúde (OMS), aproximadamente 40\% da população mundial adulta encontrava-se com sobrepeso e $13 \%$ eram obesos, no ano de 2016. Assim, foi evidenciado que a prevalência global da obesidade quase triplicou desde 1975. Em relação ao Brasil, no mesmo ano, um pouco mais da metade da população adulta estava categorizada como sobrepeso e, aproximadamente, um quinto dos adultos como obesos.

Além disso, associado à obesidade abdominal, existem outros distúrbios fisiológicos e metabólicos, que intensificam o risco de desenvolver doenças crônicas não transmissíveis, com mortalidade estimada, no ano de 2016, de 975,4 mil pessoas no Brasil (WORLD HEALTH ORGANIZATION, 2018).

Nesse contexto, a síndrome metabólica (SM) possui destaque específico por se caracterizar como um conjunto de fatores de risco cardiovascular (RCV) bem definido que juntos amplificam a taxa de mortalidade e morbidade cardiovascular (NILSSON PM, et al., 2019). Essa síndrome envolve a resistência insulínica dos tecidos orgânicos levando ao aumento dos níveis da glicemia sérica com modificações no metabolismo dos carboidratos, e, ainda, a alterações metabólicas dos lipídeos e aumento da pressão arterial (PA).

Esses fatores associados alteram a fisiologia da resposta imune, provocando um ciclo vicioso de inflamação crônica responsável pelos desfechos adversos das doenças cardiovasculares (DCV). A SM aumenta a mortalidade geral, em cerca de, 1,5 vezes e a cardiovascular próximo de 2,5 vezes (BORTOLETTO MSS, et al., 2016; BRASIL, 2005).

Assim, as doenças cardiovasculares são as patologias crônico-degenerativas que mais causam morbimortalidade na atualidade, sendo a principal causa global com mortalidade aproximada em 370 mil pessoas no ano de 2016. No Brasil, $28 \%$ do total de óbitos foram por doenças cardiovasculares, gerando gasto direto total com internações e consultas por DCV em torno R $\$ 5,1$ bilhões no ano de 2015 (WORLD HEALTH ORGANIZATION, 2018; SIQUEIRA ASE, et al., 2017). 
Com o propósito de definir a SM, muitas organizações dedicaram-se a caracterizá-la, entre elas, a OMS, a International Diabetes Federation (IDF) e a American Association of Clinical Endocrinólogists (AACE), porém a mais comumente usada pela Sociedade Brasileira de Cardiologia, por ter sido desenvolvida para uso clínico, é a da National Cholesterol Education Program (NCEP/ATPIII) que define a SM pela existência de três dentre cinco fatores básicos estabelecidos que são: hipertrigliceridemia, obesidade abdominal, lipoproteina de alta densidade (HDL) baixo, hipertensão arterial sistêmica (HAS), glicemia de jejum (GJ) elevada ou, ainda, a presença de diabetes mellitus 2 (DM) (BRASIL, 2005; NILSSON PM, et al., 2019).

A SM possui prevalência elevada nos países desenvolvidos, variando segundo etnia, raça e gênero (MORBACH C, et al., 2019; NILSSON PM, et al., 2019; WOOTEN T, et al., 2019; KHOSRAVIPOUR M, et al., 2019). Em idosos, no Brasil, foi encontrada uma prevalência maior nas mulheres (40\%) do que nos homens (22\%) (PAULA JAT, et al., 2015).

A SM pode estar associada ao envelhecimento em alguns casos, devido a mesma, estar relacionada com a prevalência aumentada de alguns fatores de risco cardiovascular que compõem o diagnóstico de SM e levam a desfechos adversos, como, infarto agudo do miocárdio e acidente vascular encefálico. Dentre esses fatores de risco, a HAS possuiu prevalência de $80 \%$ em idosos da zona leste de São Paulo no ano de 2015, seguidos da hipercolesterolemia e excesso de peso, em ordem decrescente, com uma porcentagem maior para as mulheres (SIQUEIRA ASE, et al., 2017; SOAR C, 2015).

Dessa forma, tendo em vista a prevalência da SM na população idosa, o aumento da expectativa de vida associada a maior probabilidade de desfechos adversos e o seu peso nos cofres públicos, a estratificação do risco cardiovascular dessa população alvo potencializará dados úteis para a promoção à saúde e o tratamento adequado, além da reabilitação e prevenção de agravos dos portadores dessa síndrome. Diante do exposto, emerge-se o seguinte questionamento: o risco cardiovascular do paciente idoso portador de SM estará classificado em moderado/alto risco? Com isso, este trabalho visa estratificar o risco cardiovascular de pacientes idosos portadores de SM.

\section{MÉTODOS}

O presente estudo trata-se de uma pesquisa de campo do tipo quantitativa e descritiva, realizada com idosos, usuários do Sistema Único de Saúde, nas Unidades Básicas de Saúde (UBS) em uma cidade do interior da Paraíba. A estrutura de saúde da cidade funciona como microrregião de saúde e possui quarenta e uma UBS, um centro de especialidades, uma Unidade de Pronto Atendimento, e três Hospitais na abrangência de seu território.

A população idosa do município é constituída por 11.184 habitantes e foi adotada amostra probabilística aleatória simples de 178 usuários que concordaram em participar da pesquisa e que assinaram o Termo de Consentimento Livre Esclarecido, dividindo-se equitativamente os participantes pelas UBS. O número de amostra foi calculado de acordo com calculadora amostral clínica. Tendo, pois, uma maior adesão da população a pesquisa, foi totalizada com 182 participantes (SANTOS GEO, 2017).

Os critérios de inclusão para participação na pesquisa foram: possuir mais que 60 anos de idade e residir em uma cidade do estado da Paraíba. Já os critérios de exclusão da participação na pesquisa foram: não estar em jejum de 12 horas e possuir qualquer tipo de lesão no local da punção periférica.

Após aprovação do Comitê de Ética e Pesquisa, no CAEE (80780117.1.0000.5181) e no do parecer (2.438.533), foram realizadas visitas às UBS, onde os usuários aleatoriamente selecionados por microárea de abrangência foram informados através dos agentes comunitários de saúde sobre o dia da coleta de dados para a pesquisa.

$\mathrm{Na}$ data acordada, os usuários que concordaram em participar do estudo compareceram a UBS para que fosse realizada a coleta de sangue venoso periférico para a análise laboratorial de glicemia de jejum (GJ) através do método de espectrofotometria e perfil lipídico, além de medidas da circunferência abdominal (CA), do peso, da estatura e dos níveis de PA. Foi utilizado, também, um formulário para avaliar o estilo de vida da população em estudo, relacionando os fatores de risco para SM. 
Como instrumento de coleta de dados foi utilizado um formulário contendo todos os parâmetros antropométricos e laboratoriais a serem avaliados para o diagnóstico de SM segundo os critérios da NCEP/ATPIII, utilizado internacionalmente, além de dados gerais, como: idade e sexo, dados sobre o estilo de vida, incluindo hábitos alimentares, prática de exercício físico e tabagismo, e ainda, dados sobre antecedentes pessoais patológicos, como: DM, HAS, dislipidemias e história de evento cardiovascular e medicações em uso. Além disso, foram integradas informações acerca de antecedentes familiares e o conhecimento do usuário sobre a SM (BRASIL, 2005).

Os dados da amostra foram analisados utilizando software IBM SPSS Statistics 21 para correlação de variáveis através dos testes t, Qui- Quadrado, exato de Fisher e Kruskal Wallis (IC=95\%), diagnóstico dos pacientes portadores da SM conforme os critérios da NCEP/ATPIII e Estratificação do RCV segundo o Escore de Framingham (EF).

Esse score é calculado através da atribuição de pontos, pré-estabelecidos segundo o sexo, com relação às variáveis: idade, LDL-C, HDL-c, pressão arterial sistólica e diastólica, presença de DM e tabagismo que foram somados algebricamente com o total de pontos indicando em porcentagem 0 risco de evento cardiovascular em 10 anos conforme o sexo, posteriormente categorizados em baixo $(<10 \%)$, moderado (10 - 20\%) e alto risco (>20\%) cardiovascular (BRASIL, 2006).

\section{RESULTADOS}

Os fatores de risco individuais e as caraterísticas demográficas para SM dos 182 integrantes da pesquisa estão apresentados de forma concisa na Tabela 1. A média de idade dos participantes foi de 70,28 $\pm 7,14 \mathrm{e}$ variou de 60 a 92 anos, sendo $24,2 \%$ do sexo masculino e $75,8 \%$ do sexo feminino, desses, apenas $10,98 \%$ faziam o uso do tabaco. A média da chance de ocorrência de um acidente vascular cerebral (AVC) ou infarto agudo do miocárdio (IAM) (RCV) em 10 anos foi de 15,88 \pm 9,78\%.

As porcentagens para os fatores de risco da SM foram: $81,86 \%$ para PA elevada, $66,48 \%$ para CA aumentada, 59,89\% para hipertrigliceridemia, 42,85\% para GJ alterada e 32,41\% para níveis baixos de HDLc. No grupo masculino, as variáveis mais alteradas foram a PA, GJ e TG. Já no grupo feminino, as variáveis que mais se apresentaram alteradas foram CA, PA e TG. Contudo, apenas HDL-c, GJ e CA possuíram diferença estatisticamente significativa entre os sexos para as variáveis mencionadas.

Tabela 1 - Fatores de Risco para Síndrome Metabólica.

\begin{tabular}{|c|c|c|c|c|c|c|c|c|c|}
\hline \multirow[t]{2}{*}{ Variável } & \multirow[t]{2}{*}{ Categoria } & \multicolumn{2}{|c|}{$\begin{array}{l}\text { Masculino } \\
(n=44)\end{array}$} & \multicolumn{2}{|c|}{$\begin{array}{c}\text { Feminino } \\
(n=138)\end{array}$} & \multicolumn{2}{|c|}{$\begin{array}{c}\text { Total } \\
(n=182)\end{array}$} & \multirow[t]{2}{*}{$\mathrm{X}^{2}$} & \multirow[t]{2}{*}{$\mathbf{P}$} \\
\hline & & $\mathbf{N}$ & (\%) & $\mathbf{N}$ & (\%) & $\mathbf{N}$ & (\%) & & \\
\hline \multirow{2}{*}{$\begin{array}{l}\text { CA } \\
\text { (cm) }\end{array}$} & $\begin{array}{l}>102(\mathrm{M}) \\
> \\
>\end{array}$ & 15 & 34,10 & 106 & 76,82 & 121 & 66,48 & \multirow{2}{*}{27,32} & \multirow{2}{*}{$<0,01$} \\
\hline & $\begin{array}{l}\leq 102(\mathrm{M}) \\
\leq 88(\mathrm{~F})\end{array}$ & 29 & 65,90 & 32 & 23,18 & 61 & 33,52 & & \\
\hline \multirow{2}{*}{$\begin{array}{c}\mathrm{TG} \\
(\mathrm{mg} / \mathrm{dL})\end{array}$} & $\geq 150$ & 25 & 56,81 & 84 & 60,87 & 110 & 60,44 & \multirow{2}{*}{0,44} & \multirow{2}{*}{$>0,05$} \\
\hline & $<150$ & 18 & 43,19 & 54 & 39,13 & 72 & 39,56 & & \\
\hline \multirow{2}{*}{$\begin{array}{c}\text { PA } \\
(\mathrm{mmHg})\end{array}$} & $\geq 130 / 85$ & 40 & 90,91 & 109 & 78,98 & 149 & 81,87 & \multirow{2}{*}{3,19} & \multirow{2}{*}{$>0,05$} \\
\hline & $<130 / 85$ & 4 & 9,09 & 29 & 21,02 & 33 & 18,13 & & \\
\hline \multirow{2}{*}{$\begin{array}{c}\text { GJ } \\
(\mathrm{mg} / \mathrm{dL})\end{array}$} & $\geq 110$ & 26 & 59,09 & 52 & 37,68 & 78 & 42,86 & \multirow{2}{*}{6,24} & \multirow{2}{*}{$<0,05$} \\
\hline & $<110$ & 18 & 40,91 & 86 & 62,32 & 104 & 57,14 & & \\
\hline \multirow{2}{*}{$\begin{array}{c}\mathrm{HDL} \\
(\mathrm{mg} / \mathrm{dL})\end{array}$} & $\begin{array}{l}<40(\mathrm{M}) \\
<50(\mathrm{~F})\end{array}$ & 8 & 18,18 & 50 & 36,24 & 58 & 31,87 & \multirow{2}{*}{5,01} & \multirow{2}{*}{$<0,05$} \\
\hline & $\begin{array}{l}\geq 40(\mathrm{M}) \\
\geq 50(\mathrm{~F})\end{array}$ & 36 & 81,82 & 89 & 63,76 & 124 & 68,13 & & \\
\hline
\end{tabular}

Legenda: $C A$, circunferência abdominal; F, feminina; GJ, glicose plasmática em jejum; HDL-C, colesterol de lipoproteína de alta densidade; $M$, masculino; PA, pressão arterial; TG, triglicerídeos.

Fonte: RODRIGUES VR, et al. 2020. 
A prevalência da SM e seus fatores de risco estão listados na Tabela 2. Foram diagnosticados 126 $(69,23 \%)$ participantes como portadores da SM, sendo $13,75 \%$ do sexo masculino e $55,49 \%$ do sexo feminino. Comparando-se os valores de cada fator de risco para a SM, foi observado que portadores da SM possuem níveis de CA maiores em 10,51 cm, para homens, e 9,48 cm, para mulheres que indivíduos não portadores da SM.

Em relação ao HDL-c, foi observado valores menores apenas nos participantes do sexo feminino, com diferença negativa de $15,52 \mathrm{mg} / \mathrm{dL}$. No que se refere à GJ, TG e pressão arterial sistólica e diastólica temos um incremento de $33,98 \mathrm{mg} / \mathrm{dL}, 102,43 \mathrm{mg} / \mathrm{dL}, 11,51 \mathrm{mmHg}$ e $4,77 \mathrm{mmHg}$, respectivamente.

Tabela 2 - Diferenças entre fatores de risco para pacientes com e sem Síndrome Metabólica.

\begin{tabular}{|c|c|c|c|c|c|c|c|}
\hline \multicolumn{2}{|c|}{ Fatores de Risco } & $\begin{array}{l}\text { CA } \\
(\mathbf{c m})\end{array}$ & $\begin{array}{c}\text { PAS } \\
(\mathrm{mmHg})\end{array}$ & $\begin{array}{c}\text { PAD } \\
(\mathrm{mmHg})\end{array}$ & $\begin{array}{c}\mathrm{TG} \\
(\mathrm{mg} / \mathrm{dl})\end{array}$ & $\begin{array}{l}\text { HDL-c } \\
(\mathrm{ma} / \mathrm{dl})\end{array}$ & $\underset{(\mathrm{ma} / \mathrm{dl})}{\mathrm{GJ}}$ \\
\hline \multirow{4}{*}{$\begin{array}{c}\text { Total } \\
(n=182)\end{array}$} & SM & $100,1 \pm$ & $147,22 \pm$ & $88,69 \pm$ & $231,69 \pm$ & $53,10 \pm$ & $131,60 \pm$ \\
\hline & $(n=126)$ & 11,9 & 22,22 & 14,28 & 134,49 & 17,91 & 58,62 \\
\hline & Sem SM & $90,64 \pm$ & $135,71 \pm$ & $83,92 \pm$ & $120,31 \pm$ & $63,29 \pm$ & $97,62 \pm$ \\
\hline & $(n=56)$ & 11,47 & 23,42 & 12,31 & 48,12 & 17,62 & 28,18 \\
\hline \multicolumn{2}{|c|}{$\mathbf{P}$} & $<0,01$ & $<0,05$ & $<0,05$ & $<0,01$ & $<0,05$ & $<0,01$ \\
\hline \multirow{4}{*}{$\begin{array}{l}\text { Masculino } \\
\qquad(n=44)\end{array}$} & SM & $102,16 \pm$ & $147,6 \pm$ & $89,6 \pm$ & $102,16 \pm$ & $54,72 \pm$ & $138,84 \pm$ \\
\hline & $(n=25)$ & 9,41 & 19,20 & 15,67 & 169,6 & 20,44 & 53,59 \\
\hline & Sem SM & $91,65 \pm$ & $142,63 \pm$ & $86,84 \pm$ & $147,07 \pm$ & $53,56 \pm$ & $112,68 \pm$ \\
\hline & $(n=19)$ & 9,72 & 24,23 & 14,16 & 68,03 & 11,90 & 41,42 \\
\hline \multicolumn{2}{|c|}{$\mathbf{P}$} & $<0,01$ & $>0,05$ & $>0,05$ & $<0,05$ & $>0,05$ & $>0,05$ \\
\hline \multirow{4}{*}{$\begin{array}{l}\text { Feminino } \\
(n=138)\end{array}$} & SM & $99,68 \pm$ & $147,12 \pm$ & $88,46 \pm$ & $231,69 \pm$ & $52,76 \pm$ & $129,81 \pm$ \\
\hline & $(n=101)$ & 12,49 & 22,99 & 13,99 & 134,49 & 17,39 & 59,91 \\
\hline & SEM SM & $90,20 \pm$ & $90,20 \pm$ & $82,43 \pm$ & $120,31 \pm$ & $68,28 \pm$ & $89,89 \pm$ \\
\hline & $(n=37)$ & 12,36 & 12,36 & 11,15 & 48,12 & 18,11 & 13,20 \\
\hline \multicolumn{2}{|c|}{$\mathbf{P}$} & $<0,01$ & $<0,01$ & $<0,05$ & $<0,05$ & $<0,01$ & $<0,01$ \\
\hline
\end{tabular}

Legenda: CA, circunferência abdominal; GJ, glicose plasmática em jejum; HDL-c, colesterol de lipoproteína de alta densidade; PAD, pressão arterial diastólica; PAS, pressão arterial sistólica; Sem SM, não portador de síndrome metabólica; SM, portador de síndrome metabólica; TG, triglicerídeos.

Fonte: RODRIGUES VR, et al. 2020.

A correlação entre as categorias de risco cardiovascular, segundo o EF, com o diagnóstico de SM está demonstrada na tabela 3. A porcentagem de participantes diagnosticados como portadores de SM e classificados como alto risco cardiovascular em relação à amostra total foi de $37,91 \%$, desses a maioria eram mulheres $(57,9 \%), 30,76 \%$ foram classificados como tendo risco moderado e $30,76 \%$ como baixo risco. Os homens apresentaram maior associação com o risco alto/moderado de desenvolvimento de doença cardiovascular $(79,54 \%)$ em relação às mulheres $(65,21 \%)$. $O$ risco não aumentou com a idade dos pacientes $(p>0,05)$.

Houve significância estatística na relação entre a presença da SM e a distribuição dos níveis de risco cardiovascular $(p<0,001)$. Os indivíduos portadores de síndrome metabólica possuíram risco de DCV 2,14 vezes maior ( $\mathrm{IC}=95 \%$ ) que os não portadores em relação ao grupo de alto risco.

Em relação ao sexo, foi identificado que o sexo feminino aumenta o RCV em 10 anos $(p<0,01)$ comparado ao sexo masculino $(3,74: 1,52)$. Já para no grupo de baixo risco, foi observado efeito protetor da ausência da $\mathrm{SM}$ no RCV ( $R R=0,43 ; \mathrm{IC}=95 \%)$. Além disso, foi identificado que possuir mais de 3 critérios diagnósticos para a SM aumentou o risco relativo de 0,66 para 1,86 vezes em relação a classificação de alto risco. 
Tabela 3 - Diferenças entre a classificação de Risco Cardiovascular entre indivíduos com e sem Síndrome Metabólica.

\begin{tabular}{|c|c|c|c|c|c|}
\hline \multicolumn{3}{|c|}{ Risco Cardiovascular } & \multirow{2}{*}{$\begin{array}{c}\begin{array}{c}\text { Baixo } \\
(<10 \%)\end{array} \\
3 \\
(12)\end{array}$} & \multirow{2}{*}{$\begin{array}{c}\begin{array}{c}\text { Moderado } \\
(\mathbf{1 0 - 2 0 \% )}\end{array} \\
6 \\
(24)\end{array}$} & \multirow{2}{*}{$\begin{array}{c}\begin{array}{c}\text { Alto } \\
(>\mathbf{2 0 \%} \%)\end{array} \\
16 \\
(64)\end{array}$} \\
\hline $\begin{array}{c}\text { Masculino } \\
(n=44)\end{array}$ & $\begin{array}{c}S M \\
(n=25)\end{array}$ & $\begin{array}{c}\mathrm{n} \\
\%)\end{array}$ & & & \\
\hline & $\begin{array}{c}\text { Sem SM } \\
(n=19)\end{array}$ & $\begin{array}{c}\mathrm{n} \\
(\%) \\
\end{array}$ & $\begin{array}{c}5 \\
(26,31)\end{array}$ & $\begin{array}{c}5 \\
(26,31)\end{array}$ & $\begin{array}{c}8 \\
(42,10)\end{array}$ \\
\hline & $\mathbf{P}$ & & $>0,05$ & $>0,05$ & $>0,05$ \\
\hline $\begin{array}{c}\text { Feminino } \\
(n=138)\end{array}$ & $\begin{array}{c}\text { SM } \\
(n=101)\end{array}$ & $\begin{array}{c}n \\
(\%)\end{array}$ & $\begin{array}{c}22 \\
(21,78)\end{array}$ & $\begin{array}{c}38 \\
(37,62)\end{array}$ & $\begin{array}{c}41 \\
(40,59)\end{array}$ \\
\hline & $\begin{array}{c}\text { Sem SM } \\
(n=37)\end{array}$ & $\begin{array}{c}\mathrm{n} \\
\%)\end{array}$ & $\frac{26}{(76,47)}$ & $\begin{array}{c}7 \\
(20,58)\end{array}$ & $\frac{4}{(11,76)}$ \\
\hline & $\mathbf{P}$ & & $<0,01$ & $<0,05$ & $<0,01$ \\
\hline $\begin{array}{c}\text { Total } \\
(n=182)\end{array}$ & $\begin{array}{c}\text { SM } \\
(n=126)\end{array}$ & $\begin{array}{c}\mathrm{n} \\
\%)\end{array}$ & $\begin{array}{c}25 \\
(19,84)\end{array}$ & $\begin{array}{c}44 \\
(34,92)\end{array}$ & $\begin{array}{c}57 \\
(45,23)\end{array}$ \\
\hline & $\begin{array}{c}\text { Sem SM } \\
(n=56)\end{array}$ & $\begin{array}{c}n \\
\%)\end{array}$ & $\frac{32}{(55,35)}$ & $\frac{12}{(21,42)}$ & $\frac{12}{(21,42)}$ \\
\hline & $\mathbf{P}$ & & $<0,01$ & $>0,05$ & $<0,05$ \\
\hline
\end{tabular}

Legenda: DCV, doença cardiovascular; Sem SM, não portador de síndrome metabólica; SM, portador de síndrome metabólica.

Fonte: RODRIGUES VR, et al. 2020.

No que se refere a CA, foi observado maior predomínio de obesidade central entre as mulheres $76,81 \%$ que entre os homens (34,09\%). A prática regular de exercício físico era realizada por $41,1 \%$ dos indivíduos, destes, o tipo caminhada foi o mais prevalente $(89,33 \%)$ comparado à hidroginástica $(5,88 \%)$, musculação $(2,66 \%)$ e pilates $(1,33 \%)$. A aplicação do teste $x^{2}$ não evidenciou significância estatística desses parâmetros correlacionados à SM.

Das classificações de índice de massa corpórea (IMC) encontradas nos pacientes, o sobrepeso possuiu maior prevalência $(57,1 \%)$ em relação ao adequado $(28,6 \%)$ e baixo peso $(14,3 \%)$. Além disso, existiu correlação linear não significativa $(p>0,05)$ entre o risco de DCV em 10 anos e os valores do IMC.

\section{DISCUSSÃO}

O presente estudo abordou a prevalência do risco de DCV em 10 anos em uma população atendida pelo serviço de saúde pública municipal. Esse tipo de população pode ser reconhecido como população vulnerável por possuir maior suscetibilidade para doenças cardiovasculares devido ao seu nível socioeconômico menos favorecido (KIM C-J, et al., 2014).

Tendo em vista que a presença da SM pode aumentar com a idade para o sexo feminino, a porcentagem de indivíduos acometidos pelo agravo foi próxima de $70 \%$, superior àquela encontrada em estudos no Egito e em outro estudo no Brasil, com 55\% e 33\%, respectivamente. Constatou-se, portanto, alta prevalência da SM neste estudo (ELAZIZ KM, et al., 2014; CONCEIÇÃO RDO, et al., 2015).

O sexo feminino possuiu prevalência maior da SM com porcentagem mais elevada que outros estudos analisados (PAULA JAT, et al., 2015; JIANG B, et al., 2018). Isto pode ser justificado pela melhora dos valores séricos nos homens para os componentes da SM com avançar da idade (KIM S, SO W-Y, 2016).

Os fatores de risco da SM mais encontrados foram a PA, CA e hipertrigliceridemia que diferem de um estudo coreano de população mista (meia-idade e idoso) que identificou CA, GJ e HDL-c alterados como mais prevalentes. Esse dado é, em parte, consonante com dados obtidos na Coreia que, embora houvesse uma diminuição dos níveis de triglicerídeos, foi observado um aumento da frequência da HAS com o avançar da idade. Essa discrepância pode ser explicada pelos hábitos alimentares das distintas populações estudadas (JIANG B, et al., 2018).

Pacientes portadores de SM e HAS possuem uma maior tendência no desenvolvimento precoce de alterações hormonais e metabólicas que podem se manifestar com lesão de órgão-alvo, aumentando assim, o RCV (CATHARINA AS, et al., 2018). 
No que se refere ao sexo, os fatores de risco que mais se apresentaram nos homens foram PA, GJ e TG, dois deles, também, identificados em estudo internacional, porém em proporções diferentes (LEE $S$, et al., 2016). Assim, a PA elevada é parte de um perfil metabólico de RCV elevado e, não um fator isolado.

Já para o sexo feminino, um estudo encontrou uma porcentagem cerca de $70 \%$ para obesidade metabolicamente saudável, o oposto dos achados aqui evidenciados, havendo uma presença maior de anormalidades metabólicas em mulheres e o fator obesidade abdominal como mais prevalente com diferença percentual positiva de $42 \%$ em relação aos homens (PIMENTEL AC, et al., 2015; RAMIRES EKNM, et al., 2018).

Por conseguinte, embora a obesidade central tenha possuído certa distribuição linear com o alto risco cardiovascular, esse resultado não foi significativo estatisticamente. E, estando associada com a obesidade visceral, aquela possui aumento da atividade pró-inflamatória com elevação sérica da adiponectina que pode estar relacionada à resistência à insulina, DM, HAS, aterosclerose e aumento da mortalidade (HAMER M, et al., 2015).

Além disso, a correlação entre o estado metabólico conferido pela menopausa e os componentes da SM resultou em uma elevação nos níveis de PA e glicemia nesse grupo, sendo, portanto, a menopausa é um fator de risco independente para o aumento dessas variáveis (FONSECA ÉJNC, et al., 2018).

No contexto da influência da SM sobre o risco cardiovascular, há maior estenose coronariana e aterosclerose naqueles com SM comparados ao sem SM em análises angiográficas. Além disso, existe maior progressão da doença arterial coronariana no grupo com SM (SEO MH, et al., 2015).

Assim, o EF possui excelente recomendação pelas diretrizes clínicas na mensuração do risco cardiovascular, além de possuir significativa capacidade para assistir a equipe de saúde da atenção básica na estratificação de risco dos usuários da rede de atenção devido ao seu menor custo (FALUDI A, et al., 2017). Dessa forma, a informação cedida ao paciente sobre seu RCV proporciona o compartilhamento da responsabilidade em saúde e, ainda, a promoção do autocuidado.

O acréscimo de outro componente da SM aos seus 3 critérios diagnósticos duplicou o risco relativo para a classificação de alto risco na população estudada. Desse modo, a estratificação de RCV, segundo o EF, nos portadores da SM possibilita uma visão mais ampla do estado de saúde do usuário (SUNG K-C, et al., 2015).

No que se refere à SM e o $\mathrm{EF}$, foi observado neste estudo, diferença significantemente estatística na distribuição do risco cardiovascular influenciado pela SM (KIM C-J, et al., 2014; ELAZIZ KM, et al., 2014). Embora a SM aumente o RCV em relação à população de baixo risco, não é determinante para o alto risco. Além disso, existiu, também, diferença significativa entre baixo e moderado risco, não havendo, entretanto, entre o risco moderado e alto.

Cerca de $80 \%$ dos pacientes com SM obtiveram, pelo EF, classificação de moderado/alto risco. No entanto, valores menores foram encontrados no Egito (48\%) e na Austrália (57\%). Sendo assim, além de uma alta prevalência para a SM, a população estudada apresentou elevada porcentagem de risco cardiovascular em relação à literatura pesquisada (ELAZIZ KM, et al., 2014; BAGHERI N, et al., 2015).

Algumas análises evidenciaram RCV aumentado com a idade dos indivíduos, não sendo, porém, observado na população estudada. Talvez isso tenha ocorrido devido a população ser relativamente homogênea em relação à idade, tendo em vista, que a mesma, variou um pouco mais de 14 anos (JIANG B, et al., 2018).

Ademais, análises com hipertensos brasileiros possuíram alta prevalência $(84,3 \%)$ do risco moderado/alto, principalmente em homens (SOUSA NP, et al., 2016). Resultado inverso ao encontrado nesta pesquisa.

No que confere ao risco de ocorrência de alguma DCV, na amostra geral, os portadores de SM possuíram risco aproximadamente 2 vezes maior que os não portadores, o que é considerado dentro da faixa esperada. Porém, para o sexo feminino esse risco foi próximo de 4 vezes, quase 1,5 vezes maior que o encontrado na literatura. Além disso, é importante ressaltar que a presença da SM não só aumenta o RCV, mas, também, o risco de mortalidade por DCV em cerca de 60\% (BRASIL, 2005; SUNG K-C, et al., 2015). 
A PA e o nível de TG podem ser reduzidos a partir de modificações pronunciadas do estilo de vida. Isso é confirmado por estudos que envolvendo treinamento resistido, constataram a diminuição da CA e aumento dos níveis de HDL-c. Assim, a prática do exercício físico é capaz de adiar a instituição da SM (LEE JA, et al., 2016; ALBARELLO RA, et al., 2017).

Nesse aspecto, a orientação da prática de uma atividade física por um profissional de saúde foi capaz de promover mudança significativa no estilo de vida, além de atenuação dos parâmetros IMC, CA, PA, GJ e colesterol (LUNDQVIST S, et al., 2017). Assim, para o tipo de atividade física, o treinamento contínuo de moderada intensidade, praticados por 150 min semanais, foram tão eficazes quanto os exercícios de alta intensidade de durações distintas na convalescença da SM (RAMOS JS, et al, 2017).

\section{CONCLUSÃO}

Embora tenha havido resultados com relevância estatística e homogeneidade etária da população do estudo apresentado, é necessário apontar algumas limitações do mesmo. Dentre elas, encontram-se o tamanho da amostra, algum grau de heterogeneidade em relação ao perfil socioeconômico dos indivíduos que pode interferir indiretamente no seu status de saúde e, por fim, o número limitado de recursos disponíveis para a realização do mesmo. Tendo em vista o assunto abordado, certifica-se que aproximadamente $80 \%$ dos usuários idosos com SM atendidos na Atenção Básica deste estudo apresentaram, pelo Escore de Framingham, classificação de moderado/alto risco para doença cardiovascular. Houve uma alta prevalência dos fatores de risco utilizados tanto para o risco cardiovascular, como para a classificação da síndrome metabólica. Este resultado mostra a importância da dupla aplicação, tanto dos critérios do NCEPT/ATPIII como do Escore de Framingham nesses pacientes, a fim de promover a elaboração de estratégias em saúde eficientes na condução adequada dessa população idosa, visando a prevenção e decisão terapêutica satisfatórias ao controle das doenças cardiovasculares que nelas podem ocorrer.

\section{REFERÊNCIAS}

1. ALBARELLO RA, et al. Efeitos do treinamento resistido sobre o perfil lipídico de indivíduos com síndrome metabólica. Revista Andaluza de Medicina del Deporte, 2017; 10: 142-146.

2. BAGHERI N, et al. Community cardiovascular disease risk from cross-sectional general practice clinical data: a spatial analysis. Preventing Chronic Disease, 2015; 26: 1-12.

3. BARBALHO SM, et al. Síndrome metabólica, aterosclerose e inflamação: tríade indissociável? Jornal Vascular Brasileiro, 2015; 14: 319-327.

4. BORTOLETTO MSS, et al. Síndrome metabólica, componentes e fatores associados em adultos de 40 anos ou mais de um município da Região Sul do Brasil. Caderno Saúde Coletiva, 2016; 24: 32-40.

5. BRASIL. Ministério da Saúde. Prevenção clínica de doenças cardiovasculares, cerebrovasculares e renais. Brasília: Ministério da Saúde; 2006. p. 14-22.

6. CATHARINA AS, et al. Metabolic syndrome-related features in controlled and resistant hypertensive subjects. Arquivos Brasileiros de Cardiologia, 2018; 110: 514-521.

7. CONCEIÇÃO RDO et al. Check-up and cardiovascular risk progression: is there a room for innovation? Einstein (São Paulo), 2015; 13: 196-201.

8. ELAZIZ KM, et al. Prevalence of metabolic syndrome and cardiovascular risk factors among voluntary screened middle-aged and elderly Egyptians. Journal of Public Health, 2014; 37: 612-617.

9. FALUDI A, et al. Atualização da diretriz brasileira de dislipidemias e prevenção da aterosclerose - 2017. Arquivos Brasileiros de Cardiologia, 2017; 109: 1-76.

10. FONSECA ÉJNC, et al. Metabolic syndrome and insulin resistance by HOMA-IR in menopause. The International Journal of Cardiovascular Sciences, 2018; 31: 201-208.

11. HAMER M, et al. Stability of metabolically healthy obesity over 8 years: the English Longitudinal Study of Ageing. European Journal of Endocrinology, 2015; 173: 703-708.

12. I Diretriz Brasileira de Diagnóstico e Tratamento da Síndrome Metabólica. Arquivos Brasileiros de Cardiologia, 2005; 84: 3-28

13. JIANG B, et al. Age and gender-specific distribution of metabolic syndrome components in East China: role of hypertriglyceridemia in the SPECT-China study. Lipids in Health and Disease, 2018; 17: 1-14.

14. KHOSRAVIPOUR $M$, et al. Os efeitos do turno noturno rotativo e prolongado funcionam sobre a prevalência da síndrome metabólica e seus componentes. Diabetology \& Metabolic Syndrome, 2019; 13 (6): 3085-3089.

15. KIM C-J, et al. Prevalence of metabolic syndrome and cardiovascular risk level in a vulnerable population. International Journal of Nursing Practice, 2014; 21: 175-183.

16. KIM S, SO W-Y. Prevalence and correlates of metabolic syndrome and its components in elderly Korean adults. Experimental Gerontology, 2016; 84: 107-12. 
17. LEE JA, et al. Impact of combined lifestyle factors on metabolic syndrome in Korean men. Journal of Public Health, 2016; 39: 82-89.

18. LEE S, et al. Gender differences in metabolic syndrome components among the Korean 66-year-old population with metabolic syndrome. BMC Geriatrics, 2016; 16: 1-25.

19. LUNDQVIST S, et al. Physical Activity on Prescription (PAP), in patients with metabolic risk factors. A 6-month followup study in primary health care. PLoS ONE, 2017; 12: 1-15.

20. MORBACH C, et al. Variações nos fatores de risco cardiovascular em pessoas com e sem histórico de migração na Alemanha - Resultados do estudo de coorte STAAB. International Journal of Cardiology, 2019; 286: 186-189.

21. NILSSON PM, et al. The metabolic syndrome - What is it and how should it be managed?. European Journal of Preventive Cardiology, 2019; 26(2_suppl): 33-46.

22. PIMENTEL AC, et al. Characterization of metabolically healthy obese brazilians and cardiovascular risk prediction. Nutrition, 2015; 31: 827-33.

23. RAMIRES EKNM, et al. Prevalence and factors associated with metabolic syndrome among brazilian adult population: National Health Survey - 2013. Arquivos Brasileiros de Cardiologia, 2018; 110: 1-13.

24. RAMOS JS, et al. Low-volume high-intensity interval training is sufficient to ameliorate the severity of metabolic syndrome. Metabolic Syndrome and Related Disorders, 2017; 15: 319-328.

25. SANTOS GEO. Cálculo amostral: calculadora on-line. 2017. Disponível em: https://praticaclinica.com.br/anexos/ccolaborativa-calculo-amostral/ccolaborativa-calculo-amostral.php.

26. SEO MH, et al. Metabolic syndrome criteria as predictors of subclinical atherosclerosis based on the coronary calcium score. The Korean Journal of Internal Medicine, 2015; 30: 73-81.

27. SIQUEIRA ASE, et al. Analysis of the economic impact of cardiovascular diseases in the last five years in brazil. Arquivos Brasileiros de Cardiologia, 2017; 109: 39-46.

28. SOAR C. Prevalência de fatores de risco cardiovascular em idosos não institucionalizados. Revista Brasileira de Geriatria e Gerontologia, 2015; 18: 385-95.

29. SOUSA NP, et al. Estratificação de risco cardiovascular na atenção primária segundo Escore de Framingham. Tempus Acta Saude Coletiva, 2016; 10: 157-168.

30. SUNG K-C, et al. Increased cardiovascular mortality in subjects with metabolic syndrome is largely attributable to diabetes and hypertension in 159971 korean adults. The Journal of Clinical Endocrinology and Metabolism, 2015; 100: 2606-2612.

31. PAULA JAT, et al. Metabolic syndrome prevalence in elderly of urban and rural communities participants in the HIPERDIA in the city of Coimbra/MG, Brazil. Investigación y Educación en Enfermería, 2015; 33: $225-333$.

32. WOOTEN T, et al. O risco metabólico em idosos está associado à atenção prolongada prejudicada. Neuropsicologia, 2019; 33 (7): 947-955.

33. WORLD HEALTH ORGANIZATION (WHO). Global Health Estimates 2016: deaths by cause, age, sex, by country and by region, 2000-2016. Disponível em: https://www.who.int/healthinfo/global_burden_disease/estimates/en/index1.html. Acesso em: 14 nov. 2018.

34. WORLD HEALTH ORGANIZATION (WHO). Obesity and overweight. Disponível em: http://www.who.int/newsroom/fact-sheets/detail/obesity-and-overweight. Acesso em: 14 nov. 2018. 